\section{ECG Biometrics}

João Ribeiro Pinto and Jaime S. Cardoso

INESC TEC and Faculdade de Engenharia da Universidade do Porto, Porto, Portugal

\section{Synonyms}

Electrocardiogram-based biometrics; ECG authentication; ECG identification; ECG verification

\section{Definition}

ECG biometric recognition concerns the automatic identification of a person or the automatic verification of an identity claim (authentication) based on electrocardiographic signals.

\section{Background}

Biometric recognition is the task of identifying (or verifying the identity of) a subject based on their intrinsic characteristics. Characteristics used for biometric recognition include anatomical traits (such as face, fingerprints, iris), physiological traits (electrocardiogram, electroencephalogram, photoplethysmogram), and behavioral traits (such as gait, signature, or voice) (Jain et al. 2011).
The electrocardiogram (ECG) can be characterized as a physiological biometric trait and belongs to the subcategory of medical traits, alongside the electroencephalogram (EEG), the electromyogram (EMG), and the photoplethysmogram (PPG). Just like these traits, the ECG has initially been used mainly for medical diagnosis, since this signal includes valuable information on the heart structure and operation (Pinto et al. 2018).

The use of ECG signals for biometric recognition was first hypothesized by Forsen et al. (1977), who highlighted its promising capabilities among 33 potential biometric traits. In fact, several researchers have since praised the ECG for its universality, permanence, distinctiveness, and performance benefits relative to other traits (Abo-Zahhad et al. 2014).

The first research works entirely focused on ECG as a biometric trait were conducted by Biel et al. (2001) and Kyoso and Uchiyama (2001), which confirmed the potential of these signals for human recognition. These pioneering works were built around highly controlled medical acquisitions (called on-the-person settings), characterized by high signal-to-noise ratio and tight intrasubject trait variability. Hence, recognition approaches were simpler, mainly focused in fiducial ECG features and holistic heartbeat waveform matching.

Lately, the focus has shifted toward onthe-person settings, aiming to more closely approximate ECG signal quality in real unconstrained biometric applications. The use 
of fewer electrodes, only in contact with the hands and wrists, and the acquisition from subjects performing tasks results in significantly increased noise and variability. To deal with these hurdles, more sophisticated approaches based on deep learning are quickly replacing traditional methods.

\section{Theory}

The electrocardiogram results from the heart's activity. To fulfil its goal of pumping blood throughout the body, the heart needs to contract its muscle tissue, the myocardium, repeatedly and in a very coordinated fashion. The contraction of the muscle is caused by the electrical depolarization of its cells, which follows a welldefined path from the sinoatrial node (where the action potential is generated) throughout the myocardium.

The electrical currents caused by the depolarization and subsequent repolarization of the myocardium cells can be measured, resulting in the electrocardiographic signal (ECG). This signal is composed of a cyclical repetition of five easily recognizable waveforms $(\mathrm{P}, \mathrm{Q}, \mathrm{R}, \mathrm{S}$, and $\mathrm{T})$ that compose a heartbeat and are linked to specific moments of the depolarization flow (see Fig. 1). The Q, R, and $S$ waveforms compose the QRS complex, highly used for ECG biometrics since it is less affected by heart rate variability (Pinto et al. 2018).

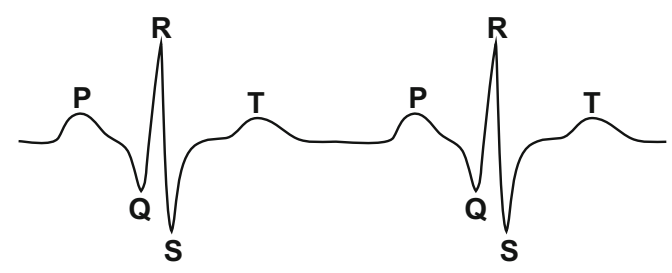

ECG Biometrics, Fig. 1 Two heartbeats of an example Lead I ECG signal illustration, with the waveforms P, $\mathrm{Q}, \mathrm{R}, \mathrm{S}$, and T. The amplitude and duration of these waveforms, although influenced by emotional states and physical activity, are where the ECG carries its identity information
For medical applications, the ECG is commonly acquired from subjects at rest, using several gel electrodes distributed across the chest and limbs in predefined places. For biometrics, off-the-person settings are the preferred way to acquire ECG, using fewer (2-3) dry electrodes in contact with the hands or wrists of the subject.

What makes the ECG suitable for biometrics is how its waveforms vary with user-specific characteristics, most notably the geometry of the heart, on their amplitude and duration, as well as the distance between fiducial points. This intersubject variability is what confers the ECG information on identity (Hoekema et al. 2001).

On the other hand, the amplitude and duration of waveforms can be influenced by intrasubject variability factors, unrelated to identity, including illnesses, emotions, stress, or drowsiness (Schijvenaars 2000). These factors distort the identity information carried by the ECG and making it more difficult to distinguish between individuals.

The main goal of an ECG-based biometric recognition system is to attenuate the effects of intrasubject variability, emphasizing the effects of intersubject variability, to make it easier to discriminate between subjects. Hence, most algorithms follow a common structure composed of signal denoising, signal preparation (involving segmentation, normalization, and outlier detection), feature extraction, and decision (see Fig. 2). As research in the topic delves further into deep learning methodologies, the compliance with this common structure is becoming increasingly feeble.

\section{Applications}

ECG biometrics finds its key applications where stronger traits (such as face or fingerprint) are unreliable or their acquisition is unfeasible, but near-continuous contact with electrodes is possible. Human-machine interface scenarios such as driving a vehicle or using a desktop comprise some of these applications. Here, the unconstrained movement of users places difficulties in the reliable measurement of other traits, but the almost continuous contact with the machine 


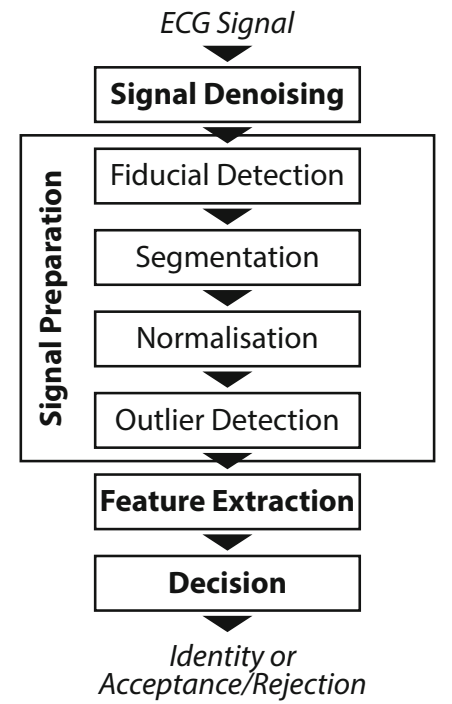

ECG Biometrics, Fig. 2 General structure of an ECGbased biometric recognition algorithm. As traditional approaches are forfeited in favor of deep learning methodologies, different processes have recently been combined in single models for enhanced performance

makes it possible to acquire the ECG signals uninterruptedly. In cases where ECG acquisition is also faulty, the combination of ECG with another trait in a multimodal biometric system can offer improved reliability.

Most notably, the use of ECG as a biometric trait opens up new opportunities on soft biometrics, especially those related to health and well-being. As a physiological signal, the ECG contains much more information on emotions, stress, and illnesses than other traits like face or fingerprint. Considering the low processing needs of the 1D ECG signals, relative to 2D, 2.5D, 3D, or 4D traits, it makes it easier to perform soft biometric recognition alongside identity recognition, continuously and in real time.

Currently, commercial applications of ECG biometrics can be found mainly in smartwatches and smart wristbands. Most are focused in the measurement of heart rate and detection of arrhythmias, but there are alternatives focused on the user identity verification using the ECG. In research, several prototypes have been developed for ECG acquisition and biometric recognition using electrodes in smartphone covers, car seats, steering wheels, computer keyboards, television remote controls, and gamepads. All these reflect the philosophy of making the acquisition as unconstrained and seamless as possible.

\section{Open Problems and Future Directions}

Currently, one of the three major open problems in ECG biometrics is the extent of the intrasubject variability factors' impact on the signal. In realistic conditions, with signals acquired in off-theperson settings, the ECG signal suffers significant influence of noise and variability, which seriously degrades biometric performance. Even with medical acquisition settings, which result in cleaner signals, the ECG still presents reduced temporal stability which leads to significant performance decay over long-term periods.

This issue is partially clouded by the second major open problem: the unrealistic evaluation settings. In the literature, the common evaluation setups only use data acquired in on-theperson settings, with lesser noise and variability influence. This translates into overly optimistic performance results that are not verifiable in real applications and draws attention away from the most relevant current issue in ECG biometrics.

The third major problem, to some extent a cause for the second one, is the lack of adequate public data. As with any other pattern recognition problem, data is of utmost importance in ECG biometrics. Currently, there is plenty of publicly available data in on-the-person settings, most notably in the PhysioNet repository. However, when addressing problems related to offthe-person noise or long-term variability, there are very few databases that offer an adequate platform for the development of more robust algorithms.

In the future, one should expect to see the development of more complete public databases and the emergence of more sophisticated and robust algorithms for ECG biometrics. These should be accompanied by novel techniques to acquire ECG signals seamlessly, in even more comfortable, unconstrained, and reliable ways. Ideally, the future would see the creation of ECG 
biometric systems that continuously monitor the identity and health of the users, almost without their knowledge, with performance levels similar or superior to current major biometric traits.

\section{Cross-References}

- Authentication

$\checkmark$ Biometrics

$\checkmark$ Identification

- Identity Verification

\section{References}

Abo-Zahhad M, Ahmed SM, Abbas SN (2014) Biometric authentication based on PCG and ECG signals: present status and future directions. Signal Image Video Proces 8(4):739-751. https://doi.org/10.1007/ s11760-013-0593-4

Biel L, Pettersson O, Philipson L, Wide P (2001) ECG analysis: a new approach in human identification. IEEE Trans Instrum Meas 50(3):808-812. https://doi.org/10. $1109 / 19.930458$
Forsen GE, Nelson MR, Staron Jr RJ (1977) Personal attributes authentication techniques. Technical report, Pattern Analysis and Recognition Corporation, Rome Air Development Center New York

Hoekema R, Uijen GJH, van Oosterom A (2001) Geometrical aspects of the interindividual variability of multilead ECG recordings. IEEE Trans Biomed Eng 48(5):551-559. https://doi.org/10.1109/10.918594

Jain AK, Ross AA, Nandakumar K (2011) Introduction to biometrics. Springer Publishing Company, Incorporated

Kyoso M, Uchiyama A (2001) Development of an ECG identification system. In: Engineering in medicine and biology society, 2001. Proceedings of the 23rd annual international conference of the IEEE, vol 4, pp 37213723. https://doi.org/10.1109/IEMBS.2001.1019645

Pinto JR, Cardoso JS, Lourenço A (2018) Evolution, current challenges, and future possibilities in ECG biometrics. IEEE Access 6:34746-34776. https://doi. org/10.1109/ACCESS.2018.2849870

Schijvenaars RJA (2000) Intra-individual variability of the electrocardiogram: assessment and exploitation in computerized ECG analysis. Ph.D. thesis, Erasmus University Rotterdam 